\title{
STRUCTURE OF THE INNER GALACTIC DISK AND THE BULGE - FIRST RESULTS
}

\author{
S. FELTZING \\ RGO \& IoA, Cambridge, UK
}

Is there an age and/or a metallicity gradient in the Bulge? This is a notoriously difficult question because of the well known age-metallicitydistance degeneracy in colour magnitude diagrams (CMD) as well as the severe crowding and large reddening towards the Galactic Bulge. The current observational data on the bulge in our galaxy and bulges in other spiral galaxies point in disparate directions, that is evidence for both early (e.g. existence of very old halo and bulge globular clusters) and late formation (e.g. Sgr dSph and bar instabilities) can be found as well as the existence and non-existence of metallicity gradients (for a review of the observational status see Wyse, Gilmore \& Franx 1997). We here present the CMDs for two fields, Baade's window and SGR-I, Fig.1c and d. Both these regions have low extinction. To determine the age and metallicity for these stars we compare the CMDs with CMDs of globular clusters, also observed with WFPC2, of known metallicity and age, Fig.1a and b. This method enables us to work entirely in the in-flight magnitude system of WFPC2 and there is no need for transformations to standard colours and magnitudes, something which is not straight forward for WFPC2 passbands.

The archival observations we have used for the clusters have previously been reported in Ortolani et al. (1995). We have derived our own photometry from the WFPC2 images. For NGC6553 our CMD clearly shows stars that appears to be trace out a giant branch redder than that of the cluster itself and the turn-off of a background population, at $V \sim 21^{m}-21^{m} .5$. The reason for these features being clearly visible in our CMD appears to stem from the fact that we have been able to extract magnitudes with smaller errors at $V \sim 20^{m}$ then Ortolani et al. (1995). We propose that the second giant branch is in fact the giant branch of the galactic bulge and not the effect of differential reddening (see Ortolani et al. 1995 and references therein) and that the turn-off is the turn-off of the Bulge. NGC6553 is sit- 

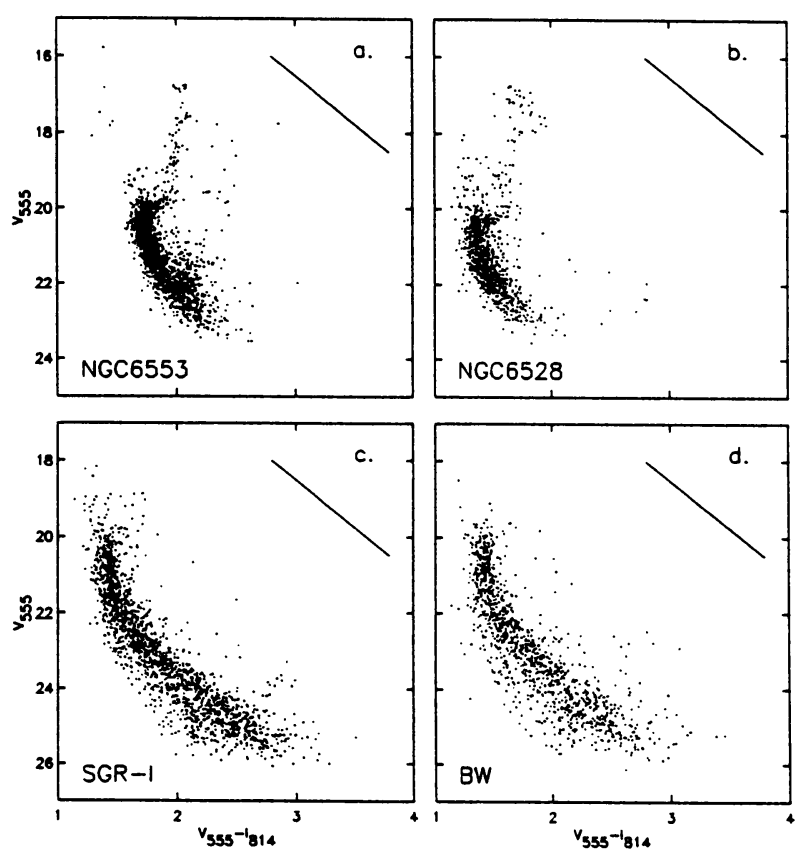

Figure 1. CMDs derived from WFPC2 PC1 images. The magnitudes are in the HST flight system (F555W and F814W) and calibrated as described in Holtzman et al. (1995). The faintest stars have errors less than $0^{m} .1$ in $I_{814}$ and less than $0^{m} .12$ in $V_{555}$, brighter stars have smaller errors. The direction of the reddening vector is indicated by a solid line. SGR-I $(1, b)=(1.27,-2.66), 1722$ stars detected, BW $(1.14,-3.77), 1149$ stars, NGC6528 $(1.14,-4.17), 998$ stars, NGC6553 $(5.25,-3.02), 1666$ stars.

uated roughly $4.8 \mathrm{kpc}$ from the Sun and thus $\sim 3 \mathrm{kpc}$ from the galactic centre. The position of the second turn-off is consistent with a Bulge turnoff at $M_{V} \sim 3$, a distance modulus of $\sim 14^{m} .5$, an extinction towards the cluster of $\sim 3^{m}$ in F555W (Ortolani et al. 1990) and a further extinction of roughly $1^{m}$ towards the Bulge.

It seems plausible that the wider main sequences in the SGR-I and BW CMDs, as compared to the cluster CMDs, are a result of a metallicity spread in the Bulge convolved with a depth effect. The reddening for SGR-I, BW and NGC6528 are very similar, thus the turn-off colours imply that the age of oldest Bulge population is not much different from that of the cluster, provided they have similar metallicities.

\section{References}

Holtzman et al., 1995 PASP, 107, 1065

Ortolani S., Renzini A., Gilmozzi R., Marconi G., Barbuy B., Bica E., Rich M., 1995, NATURE, 377, 701

Wyse, R.F.G., Gilmore G., Franx M., 1997, ARA\&A, 35, 637 